University of Nebraska - Lincoln

DigitalCommons@University of Nebraska - Lincoln

May 2003

\title{
Studies of magnetic properties of the stabilizing layer for synthetic antiferromagnetically coupled media
}

\author{
Z.S. Shan \\ Komag Inc., San Jose, California \\ L. Bitman \\ Komag Inc., San Jose, California \\ S.S. Malhotra \\ Komag Inc., San Jose, California \\ D. Stafford \\ Komag Inc., San Jose, California \\ B. Bian \\ Komag Inc., San Jose, California \\ See next page for additional authors
}

Follow this and additional works at: https://digitalcommons.unl.edu/physicssellmyer

Part of the Physics Commons

Shan, Z.S.; Bitman, L.; Malhotra, S.S.; Stafford, D.; Bian, B.; and Bertero, G., "Studies of magnetic properties of the stabilizing layer for synthetic antiferromagnetically coupled media" (2003). David Sellmyer Publications. 30.

https://digitalcommons.unl.edu/physicssellmyer/30

This Article is brought to you for free and open access by the Research Papers in Physics and Astronomy at DigitalCommons@University of Nebraska - Lincoln. It has been accepted for inclusion in David Sellmyer Publications by an authorized administrator of DigitalCommons@University of Nebraska - Lincoln. 


\section{Authors}

Z.S. Shan, L. Bitman, S.S. Malhotra, D. Stafford, B. Bian, and G. Bertero 


\title{
Studies of magnetic properties of the stabilizing layer for synthetic antiferromagnetically coupled media
}

\author{
Z. S. Shan, ${ }^{\text {a) }}$ L. Bitman, S. S. Malhotra, D. Stafford, B. Bian, and G. Bertero \\ Komag Inc., 1710 Automation Parkway, San Jose, California 95131 \\ Y. F. Xu, M. L. Yan, and D. J. Sellmyer \\ Materials for Research and Analysis, University of Nebraska, Lincoln, Nebraska 68588-0113
}

(Presented on 14 November 2002)

\begin{abstract}
The effects of the stabilizing layer thickness and its temperature dependence on the magnetic properties were investigated experimentally. These results were used to analyze the magnetic structure of the thin stabilizing layer and its effect on the coupling strength, which is valuable for improving the design of synthetic antiferromagnetically coupled media. (c) 2003 American Institute of Physics. [DOI: 10.1063/1.1555794]
\end{abstract}

\section{INTRODUCTION}

Synthetic antiferromagnetically (SAF) coupled media benefit from the stabilizing layer $\left(L_{S}\right)$, which is coupled antiferromagnetically with the magnetic recording layer. ${ }^{1-3}$ Therefore, the structural and magnetic properties of the stabilizing layer are essential in controlling the magnetic and recording performance of SAF media. There has been considerable work done to investigate the magnetic and reversal properties of SAF media, ${ }^{1-11}$ however, most of it was focused on the top magnetic layer, and the magnetic properties of $L_{S}$ and its correlation with the coupling strength $J$ have not been investigated intensively. In some of the earlier works, the magnetic properties of $L_{S}$ were treated as the parameters independent of the stabilizing layer thickness $\left(t_{S}\right)$, however this may not be true for a very thin $L_{S}$.

In this article, we have investigated the magnetic properties for the stabilizing layer in a wide thickness range. The experimental results reveal the correlation between the magnetic ordering of $L_{S}$ and the coupling strength $J$ and the effects of the stabilizing layer thickness $\left(t_{S}\right)$ and temperature $(T)$ on the magnetic properties of $L_{S}$. These results are valuable for improving the understanding of the magnetic structure of $L_{S}$ and the optimal design of SAF media.

\section{EXPERIMENTS}

Three sets of samples were prepared for this study. The film configuration for set $\mathrm{I}$ is underlayer/CoCrTaX/Ru/ $\mathrm{CoCrPtB} /$ overcoat. Set II is similar to set I except without the top magnetic layer. The thickness of the stabilizing layer $\left(t_{S}\right)$ varies from 20 to $100 \AA$ for set I and from 20 to $210 \AA$ for set II. Samples of set I and II were used to investigate the magnetization $\left(M_{S}\right)$ of $L_{S}$ in a broad $t_{S}$ region. The film configuration of set III is similar to set I except the $t_{S}$ was varied in a small increment from 17 to $60 \AA$. This set of samples was used for measurements of the effect of $t_{S}$ on the magnetic properties. The structural properties were investi-

a) Author to whom correspondence should be addressed; electronic mail: zhengsheng.shan@komag.com gated by x-ray diffraction, x-ray photoemission spectroscopy (XPS) and the magnetic properties were characterized with an alternating gradient force magnetometer.

\section{RESULTS AND DISCUSSION}

The film crystallographic texture is of critical importance for the recording media and the $\mathrm{Co}(110) / \mathrm{Cr}(002)$ texture is desirable. The $\mathrm{x}$-ray diffraction indicates that the $\mathrm{Co}(110) / \mathrm{Cr}(002)$ grows well expitaxially for all films studied. Figure 1 shows the $\theta-2 \theta$ and rocking scans of $\operatorname{Co}(110)$ peak for the stabilizing layer of set II films. Figure 1(a) shows that the $\mathrm{Co}(110)$ peak height, which has been normalized to $100 \AA$ for all three films, decreases with decreasing $t_{S}$, and that the peak position moves towards lower $2 \theta$ value as $t_{S}$ decreases. These features are attributed mainly to the stress effect in the initial growth of $\mathrm{Co}(110)$ planes and the diffusion of the $\mathrm{Cr}$ atoms from the underlayer. These two facts expand the lattice constant of the Co alloy in the interface region which causes this peak shift. The interface region with more $\mathrm{Cr}$ atoms also reduces the $\mathrm{Co}(110)$ diffraction intensity and this has a stronger effect for the thinner $L_{S}$. Figure 1(b) shows the rocking scans for these films. The full width at 50\% amplitude of the rocking curves increases gradually with decreasing $t_{S}$, however, even for the very thin $L_{S}$ of $20 \AA$ thickness the $c$ axes of the hexagonal-closepacked (hcp) cell of the Co alloy are still oriented preferably on the film plane.

Magnetization $\left(M_{S}\right)$ describes the most fundamental property for a magnetic material. The evolutions of " $M_{S}$ vs $t_{S}$ " and " $M_{S}$ vs $1 / t_{S}$ " for set I and II samples are shown in Figs. 2(a) and 2(b), respectively. It is seen that $M_{S}$ depends strongly on $t_{S}$, especially for $t_{S}<50 \AA$ [Fig. 2(a)], which is about the thickness regime of the stabilizing layer for current SAF media. Figure 2(b) indicates that the dead layer thickness is $\sim 15 \AA$ from the linear fitting of $M_{S}$ vs $1 / t_{S}$ and the intrinsic magnetization, i.e., the magnetization without the dead layer, is $554 \mathrm{emu} / \mathrm{cm}^{3}$. These results reveal that the stabilizing layer is not well ordered ferromagnetically for $t_{S}$ $<50 \AA$. This ordering can be improved significantly, if $t_{S}$ increases. However, it is not desirable to have thicker $L_{S}$ of 

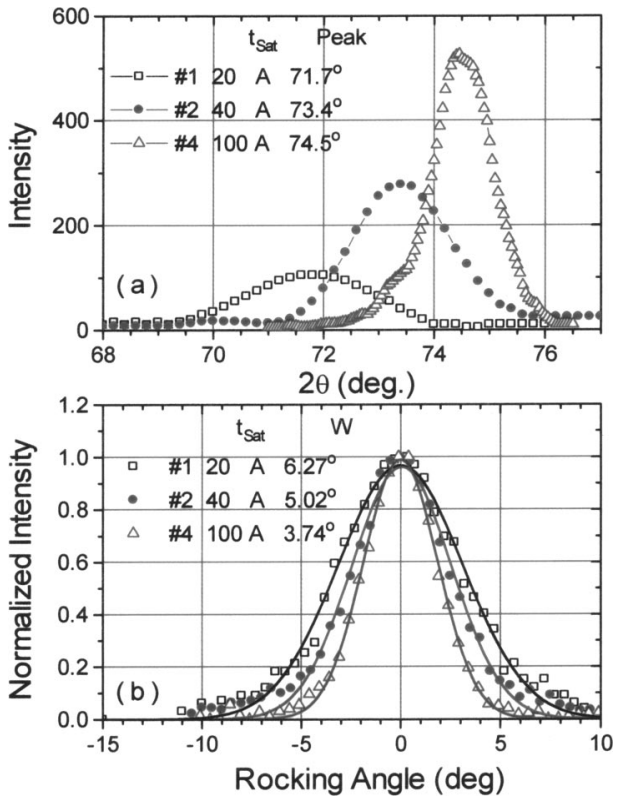

FIG. 1. X-ray diffraction pattern of $\mathrm{Co}(110)$ peaks for films with stabilizing layer thickness $t_{S}=20,40$, and $100 \AA$ : (a) x-ray $\theta-2 \theta$ scans. The diffraction intensity corresponds to $100 \AA$ thickness for all films, and (b) x-ray rocking scans.

$t_{S}>50 \AA$ for the current SAF media. This is an important issue to be considered in order to obtain more benefits from the stabilizing layer.

The magnetic properties of the stabilizing layer for a SAF film can be determined by measuring the minor loops at different $H$ field sweep rates. ${ }^{10}$ The dependence of stabilizing layer thickness on magnetization $\left(M_{S}\right)$, exchange field $\left(H_{\text {ex }}\right)$, coupling strength $(J)$, coercivity $\left(H_{C}\right)$, thermal stability factor $\left(K_{u} V^{*} / k T\right)$, and anisotropy $\left(K_{u}\right)$ for set III samples are summarized in Fig. 3. The coupling $J$ is a key feature that enables the SAF film to receive the most benefits in thermal stability from the stabilizing layer. The coupling strength $J$ can be determined by $J=M_{S} H_{\text {ex }} t_{S}$, i.e., $J$ can be calculated using the $M_{S}$ and $H_{\text {ex }}$ data as shown in Fig. 3(a). It is seen that $J \approx 0$ for $t_{S}<17 \AA$ and $J$ increases linearly with increasing $t_{S}$ for $17 \AA<t_{S}<50 \AA$. This increase in $J$ value is attributed to the increase in $M_{S}$ rather than the $H_{\mathrm{ex}}$. We notice that both $H_{\mathrm{ex}}$ and $M_{S}$ start at $t_{S} \approx 17 \AA$, the onset of the magnetic ordering for the stabilizing layer, and then the exchange field $H_{\mathrm{ex}}$ changes its value slightly while the magnetization $M_{S}$ increasing significantly as $t_{S}$ varies from 17 to $60 \AA$. This behavior has been confirmed repeatedly for several sets of samples in our labs. This result indicates that the minimum thickness of $L_{S}\left(t_{\mathrm{Min}}\right)$ should be much larger than the dead layer thickness.

The coercivity $H_{C}$ [Fig. 3(b)] and anisotropy $K_{u}$ [Fig. 3(c)] of $L_{S}$ show low values for $t_{S}<30 \AA$ and increase by about an order of magnitude as $t_{S}$ varies from 30 to $60 \AA$. This indicates that $t_{S}$ needs to be greater than $30 \AA$ for a successful stabilizing layer. The thermal stability factor $\left(K_{u} V^{*} / k T\right)$ of $L_{S}$ has fairly low values of $21-42$ as $t_{S}$ varies from 25 to $60 \AA$ [Fig. 3(c)].

It has been reported that the thin magnetic films show a strong dependence of magnetic properties on temperature
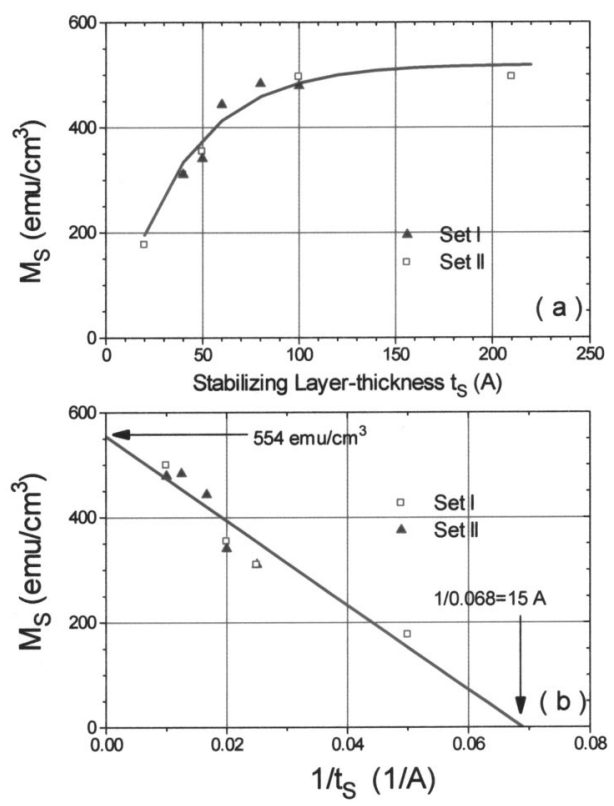

FIG. 2. Stabilizing layer thickness $\left(t_{S}\right)$ effects on magnetization $\left(M_{S}\right)$ : (a) $M_{S}$ as a function of $t_{S}$, and (b) $M_{S}$ as a function of $\left(1 / t_{S}\right)$.

(T). ${ }^{12}$ Since the $L_{S}$ thickness for the current SAF media is only about $30-50 \AA$, the temperature dependence of magnetic properties should also be investigated conscientiously. Figure 4 shows the temperature dependent behavior of magnetization $M_{S}(T)$ [Fig. 4(a)], coercivity $\left[H_{C}(T)\right]$ [Fig. 4(b)], and anisotropy field $\left[H_{K}(T)\right][$ Fig. 4(c) $]$ for a sample from set II with $t_{S}=50 \AA$. The $M_{S}(T)$ decreases by $\sim 30 \%$ as $T$ changes from $-80^{\circ} \mathrm{C}$ to $+80^{\circ} \mathrm{C}$ and the temperature effects on coercivity $H_{C}(T)$ and anisotropy $K_{u}(T)$, which can be

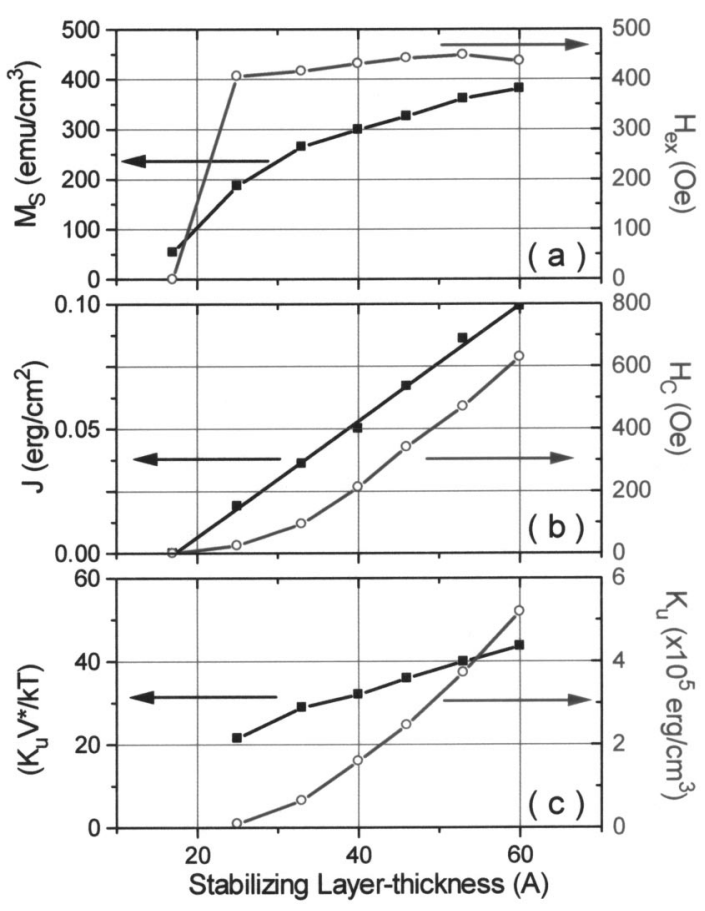

FIG. 3. Stabilizing layer thickness $\left(t_{S}\right)$ effects on magnetic properties: (a) exchange field $H_{\text {ex }}$ and magnetization $M_{S}$, (b) coupling strength $J$ and coercivity $H_{C}$, and (c) thermal stability factor $\left(K_{u} V^{*} / k T\right)$. 


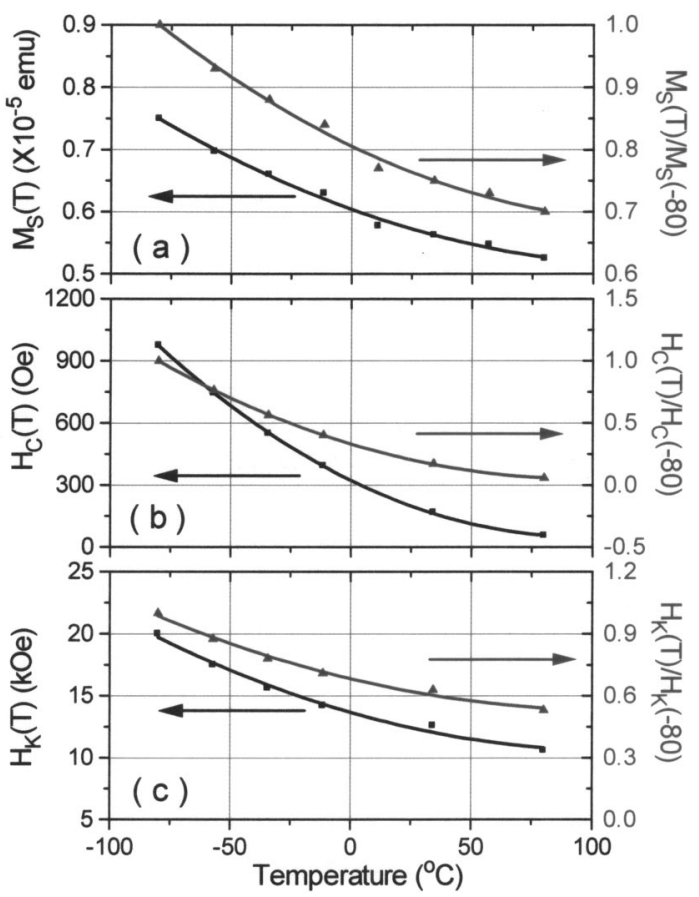

FIG. 4. Temperature effects on the magnetic properties: (a) magnetization $M_{S}(T)$, (b) coercivity $H_{C}(T)$, and (c) anisotropy field $H_{K}(T)$. The normalized values are shown on the right ordinates.

estimated by $K_{u}(T)=M_{S}(T) H_{K}(T) / 2$, are much more drastic than that of $M_{S}(T)$. This implies that temperature dependence of magnetization and coercivity, etc., should also be considered for the selection of stabilizing layer.

The appreciable effects of $t_{S}$ and $T$ on the magnetic properties for the thin stabilizing layer are correlated closely with the interdiffusion of $\mathrm{Cr}$ atoms into the stabilizing layer from the underlayer. A set of XPS profiles of $\mathrm{Co}, \mathrm{Cr}$, and C are in Fig. 5 showing the profile feature semiquantitatively. It is seen that the $\mathrm{Cr}$ atoms from the underlayer diffuse throughout the whole $L_{S}$ [Fig. 5(a)] for $t_{S}=20 \AA$, and through about half of the $L_{S}$ [Fig. 5(b)] for $t_{S}=40 \AA$, and the $\mathrm{Cr}$ atoms from the underlayer are limited in the interface or boundary region [Fig. 5(c)] for $t_{S}=100 \AA$. Thus, the diffusion of $\mathrm{Cr}$ at the interface region has a significant effect on the magnetic properties for the thinner stabilizing layer.

In summary, it has been found that a very thin stabilizing layer is not well ordered magnetically and this ordering can be improved significantly for $t_{S}>50 \AA$, which is about the upper-limit $t_{S}$ value for the current SAF media. The coupling strength $J$ increases linearly as the stabilizing layer thickness varies from 17 to $60 \AA$. The rather strong temperature and

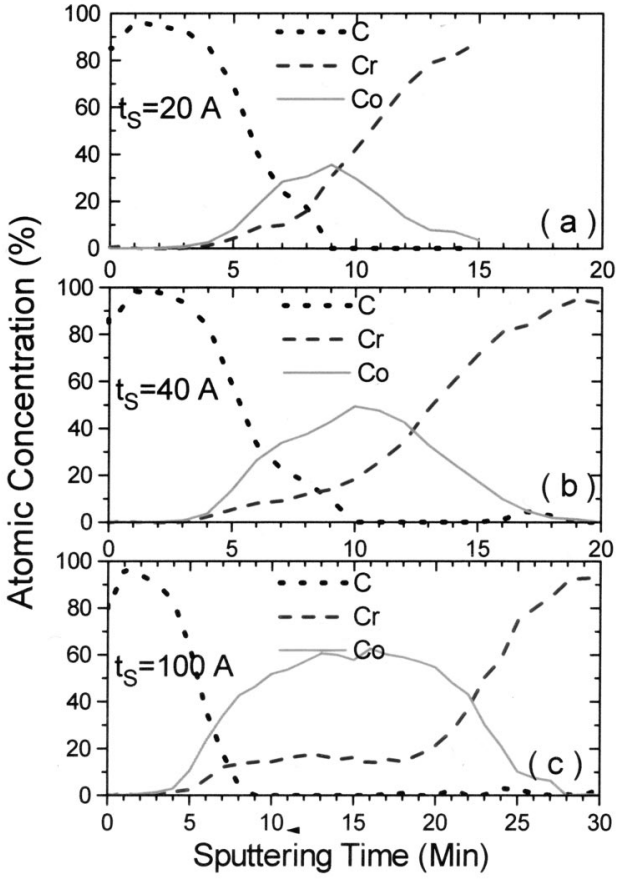

FIG. 5. The composition profiles of stabilizing layers with thickness $t_{S}$ $=20,40$, and $100 \AA$. The profile curves were measured by x-ray photoemission spectroscopy.

layer thickness effects on the magnetic properties of the stabilizing layer have to be considered conscientiously in order to improve the properties of SAF media.

${ }^{1}$ E. N. Abarra, A. Inomata, H. Sato, I. Okamoto, and Y. Mizohita, Appl. Phys. Lett. 77, 2581 (2000).

${ }^{2}$ E. E. Fullerton, D. T. Margulies, M. E. Schabes, M. Carey, B. Gurney, A. Moser, M. Best, G. Zeltzer, K. Rubin, H. Rosen, and M. Doner, Appl. Phys. Lett. 77, 3806 (2000).

${ }^{3}$ B. R. Acharya, A. Ajan, E. N. Abarra, A. Inomata, and I. Okamoto, Appl. Phys. Lett. 80, 85 (2002).

${ }^{4}$ M. Schabes, E. E. Fullerton, and D. T. Margulies, IEEE Trans. Magn. 37, 1433 (2001).

${ }^{5}$ L. Zhong, J. Giusti, and J. Fernandez-ze-Castro, IEEE Trans. Magn. 37, 1435 (2001).

${ }^{6}$ L. Guan and J. G. Zhu, IEEE Trans. Magn. 37, 1452 (2001).

${ }^{7}$ J. P. Wang, Z. S. Shan, S. N. Piramanayagam, and T. C. Chong, IEEE Trans. Magn. 37, 1445 (2001).

${ }^{8}$ G. Choe, J. N. Zhou, R. Weng, and K. E. Johson, J. Appl. Phys. 91, 7665 (2002).

${ }^{9}$ H. J. Richter and Er. Girt, Appl. Phys. Lett. 80, 2529 (2002).

${ }^{10}$ Z. S. Shan, S. S. Malhotra, D. C. Stafford, G. Betero, and D. Wachenchwanz, Appl. Phys. Lett. 81, 2412 (2002).

${ }^{11}$ Z. S. Shan, S. S. Malhotrta, D. C. Stafford, B. Bian, G. Bettero, and D. Wachenschwanz, J. Appl. Phys. 91, 7682 (2002).

${ }^{12}$ H. Zeng, Z. S. Shan, Y. Liu, M. Azarisooreh, K. Honardoost, and D. J. Sellmyer, J. Magn. Magn. Mater. 251, 283 (2002). 